\title{
STABLE MODEL EQUATIONS FOR LONG WATER WAVES
}

\author{
L. J. F. BROER, E. W. C. VAN GROESEN and J. M. W. TIMMERS \\ Dept, of Phys., Eindhoven Univ. of Technology, Eindhoven, \\ THE NETHERLANDS
}

\begin{abstract}
In this paper, a sequel to two others [1,2], some extensions and improvements of this earlier work are presented. Among these are: A more precise version of the proof of the basic canonical theorem, some considerations on conservation laws and their relation, a more complete treatment of the stability of the models, especially with respect to the wave amplitude, a short treatment of the Lagrangian version of the theory, a stable discrete model which might be useful for numerical experiments and an extension of the method to the case of slowly varying water depth.
\end{abstract}

\section{\$1. Introduction}

In two earlier papers, cited as I [1] and II [2], it was shown how the Hamilton formalism can be used to obtain satisfactory approximate equations of the Boussinesq type for fairly long, fairly low water waves. The main purpose was to find equations which are stable in the shortwave tail of the wave spectrum. The procedure used to arrive at these equations was based on the canonical theorem for the exact equations for gravity waves on an incompressible, non-viscous fluid.

The treatment as given in I and II falls short in two respects. In the proof of the canonical theorem in I some important details were omitted. In $\S 2$ we will give a more precise proof of this theorem. In deriving the approximate equations attention was focussed on short-wave instability. The possibility that solutions become unstable due to the height of the waves was not looked into. This possibility is connected with the occurrence of a term

$$
T_{f}=\int \mathrm{d} x \frac{1}{2} \eta \phi_{x}^{2}
$$

in the Hamiltonian (2.6) in II. For solutions where the motion would tend to concentrate in regions of less than average depth, that is of nega- 
tive $\eta$, (1) would be negative. In $\S 4$ we will show that this can be compensated by adding a positive term of higher order.

In the following sections we will discuss a few more examples of equations of this type. Among these will be an interesting and important set of equations due to Bona and Smith [3]. In the last section we will extend our considerations to stable approximations for waves over a sloping bottom.

\section{§ 2. The exact equations and the canonical theorem}

We start from the equations for the potential $\phi(x, y, t)$ in the dimensionless form:

$$
\begin{aligned}
& \Phi_{x x}+\Phi_{y y}=0 \\
& \Phi_{y}=0 \text { at } y=0 \\
& \left.\begin{array}{l}
h_{t}=\Phi_{y}-h_{x} \Phi_{x} \\
\Phi_{t}=-\frac{1}{2}\left(\Phi_{x}^{2}+\Phi_{y}^{2}\right)-(h-1)
\end{array}\right\} \quad \text { at } y=h(x, t) .
\end{aligned}
$$

The total energy of the fluid is:

$$
\mathscr{H}=\int \mathrm{d} x\left[\frac{1}{2}(h-1)^{2}+\int_{0}^{h} \mathrm{~d} y \cdot \frac{1}{2}\left(\Phi_{x}^{2}+\Phi_{y}^{2}\right)\right] .
$$

According to potential theory $\mathscr{H}$ is a functional of $h$ and the value of $\Phi$ at the surface when (1) and (2) are taken into account. For this surface value we write:

$$
\phi(x, t)=\Phi(x, h(x, t), t) .
$$

The canonical theorem now states that the equations of motion (3) and (4) with the constraints (1) and (2) are equivalent to the set:

$$
\begin{gathered}
h_{t}=\frac{\delta \mathscr{H}}{\delta \phi}, \\
\phi_{t}=-\frac{\delta \mathscr{H}}{\delta h} .
\end{gathered}
$$

In order to prove this we first observe that from (6),

$$
\phi_{t}=\left(\Phi_{t}\right)_{h}+\left(\Phi_{y}\right)_{h} \cdot h_{t}
$$

where the index $h$ indicates that the quantity between the brackets is to be taken at the surface $y=h$. From (7), (3) and (4) we then find:

$$
\phi_{t}=\frac{1}{2}\left(\Phi_{y}\right)_{h}^{2}-\frac{1}{2}\left(\Phi_{x}\right)_{h}^{2}-h_{x}\left(\Phi_{x}\right)_{h}\left(\Phi_{y}\right)_{h}-(h-1) .
$$


In I, $\S 2$, the variation of $\mathscr{H}$ was found to be, in the present notation:

$$
\delta \mathscr{H}=\int \mathrm{d} x\left\{\delta h\left[\left(\frac{1}{2} \Phi_{x}^{2}+\frac{1}{2} \Phi_{y}^{2}\right)_{h}+(h-1)\right]+(\delta \Phi)_{h}\left[\Phi_{y}-h_{x} \Phi_{x}\right]_{h}\right\} .
$$

From (6) we see that:

$$
(\delta \Phi)_{h}=\delta \phi-\left(\Phi_{y}\right)_{h} \delta h .
$$

Substitution of (10) in (9) then shows that (3) and (8) are indeed the canonical equations of motion. The original proof in $I$ is not essentially different from that given above. It was however rather obscure because the important relations (7) and (10) were not stated explicitly.

Finally we mention that a similar result has been given by Benjamin [4].

\section{§3. Constants of the motion}

The Hamiltonian (2.5) does not depend explicitly on the time. Therefore it must be a constant of the motion for the canonical equations:

$$
\frac{\mathrm{d}}{\mathrm{d} t} \mathscr{H}=0
$$

As our equations still are confined to the case of a horizontal bottom there is also invariance with respect to a constant variation of $x$. Therefore the canonical momentum is another constant:

$$
\frac{\mathrm{d} P}{\mathrm{~d} t}=\frac{\mathrm{d}}{\mathrm{d} t} \int \mathrm{d} x \cdot h \phi_{x}=0 .
$$

Introducing the quantity $v=\phi_{x}$ we deduce from (2.8) that

$$
\frac{\mathrm{d}}{\mathrm{d} t} \int v \mathrm{~d} x=0 \text {. }
$$

In the present context this is what is usually called a trivial constant. This means that there is no invariant canonical variation of $h$ and $\phi$ associated with (3). Nevertheless there is a variation connected with (3). In order to find it we perform a canonical transformation by replacing the pair $h, \phi$ by $v=\phi_{x}, m=\int_{x_{0}}^{x} h \mathrm{~d} x$. Obviously the Hamiltonian will be independent on the choice of $x_{0}$. That means that it is invariant with respect to the variation $\delta m=$ constant, $\delta v=0$. The related constant of the motion then is (3). 
Applying the same reasoning to the variations $\delta \phi=$ constant in the original variables we deduce that the wave volume:

$$
V=\int \mathrm{d} x(h-1), \frac{\mathrm{d} V}{\mathrm{~d} t}=0
$$

is also a constant of the motion. This is clear from the incompressibility condition. At first sight however it seems that (2.3) has not the form of a conservation law. To investigate this we consider the stream function $\Psi(x, y, t)$, defined by:

and put:

$$
\Psi_{x}=-\Phi_{y}, \quad \Psi_{y}=\Phi_{x}, \quad \Psi(x, 0, t)=0
$$

$$
\psi(x, t)=\Psi(x, h(x, t), t)
$$

It is easily seen that equation (2.3) now can be written as:

$$
h_{t}=-\psi_{x}
$$

which is a local conservation law.

The approximate equations considered in I, II and the further sections (apart from the last one) of this paper all have the same four constants of the motion, of course with the approximate Hamiltonian replacing the exact one. However, there is a relation between $P$ and $V$ which does not always hold for the approximate equations.

This relation has to do with the fact that the quantity $\psi$ is the density of the mechanical momentum:

$$
\psi=\int_{0}^{h} \mathrm{~d} y \Psi_{y}=\int_{0}^{h} \mathrm{~d} y \Phi_{x} .
$$

It is clear from (2) and (8) that the densities of the canonical and mechanical momentum differ. This difference turns out to be a derivative, therefore the momenta themselves are the same. We have, taking (8) into account:

$$
h \phi_{x}-\psi=\int_{0}^{h} \mathrm{~d} y\left(\phi_{x}-\Phi_{x}\right)=\frac{\mathrm{d}}{\mathrm{d} x} \int_{0}^{h} \mathrm{~d} y[\phi(x)-\Phi(x, y)] .
$$

Stated in other words this result means that $h$ (or $h-1$ ) is a density with conserved flux. There are two interesting consequences of this fact.

The first is a result obtained in a different way by Benjamin and Mahony [5], viz. that the center of gravity of a surface wave moves at a constant speed. We have,

$$
\frac{\mathrm{d}^{2}}{\mathrm{~d} t^{2}} \int \mathrm{d} x \cdot x h=\frac{\mathrm{d}}{\mathrm{d} t} \int \mathrm{d} x \cdot x \psi_{x}=\frac{\mathrm{d}}{\mathrm{d} t} \int \mathrm{d} x \cdot \psi=\frac{\mathrm{d} P}{\mathrm{~d} t}=0
$$


The second is the possibility of finding a relation between constants of the motion. We follow a procedure indicated in another paper [6].

We consider the quantity:

$$
C=\int \mathrm{d} x\left(t h \phi_{x}-x h\right)
$$

and calculate $\mathrm{d} C / \mathrm{d} t$ :

$$
\frac{\mathrm{d} C}{\mathrm{~d} t}=t \int \mathrm{d} x \cdot\left(h \phi_{x}\right)_{t}+\int \mathrm{d} x\left(h \phi_{x}\right)+\int \mathrm{d} x x \psi_{x}
$$

In this relation the first term vanishes on account of (2). The second and third terms cancel after integrating the latter by parts and using (9). Therefore

$$
\frac{\mathrm{d} C}{\mathrm{~d} t}=0
$$

Next we take the Poisson bracket of $C$ and some other functional $F\{h, \phi\}$. The bracket is

$$
\{C, F\}=\int \mathrm{d} x\left[\frac{\delta C}{\delta h} \frac{\delta F}{\delta \phi}-\frac{\delta C}{\delta \phi} \frac{\delta F}{\delta h}\right] .
$$

From (11) we find:

$$
\frac{\delta C}{\delta h}=t \phi_{x}-x, \frac{\delta C}{\delta \phi}=-t h_{x}
$$

therefore we have:

$$
\{C, F\}=t \int \mathrm{d} x\left(\phi_{x} \frac{\delta F}{\delta \phi}+h_{x} \frac{\delta F}{\delta h}\right)-\int \mathrm{d} x \cdot x \frac{\delta F}{\delta \phi} .
$$

We now suppose that $F$ does not depend explicitly on $x$. This gets us rid of the otherwise awkward first term. Moreover we assume that $F$ does not depend on $\phi$ itself but only on $\phi_{x}=v$ and higher derivatives. Observing that

$$
\frac{\delta F}{\delta \phi}=-\frac{\partial}{\partial x} \frac{\delta F}{\delta v}
$$

and integrating by parts finally yields:

$$
\{C, F\}=-\int \mathrm{d} x \frac{\delta F}{\delta v} .
$$


This means that, whenever the functional $F$ is a constant of the motion, $\int \mathrm{d} x \cdot(\delta F / \delta v)$ is also a constant. The operator $\{C,-\}$ converts one constant into another one. Unfortunately this operation reduces the degree of $F$ and therefore usually yields more simple constants than the starting point. We will not enter here upon the problems of inverting this operator. We only mention that for our system:

and

$$
\{C, \mathscr{H}\}=-\int \psi \mathrm{d} x=-P
$$

$$
\{C, P\}=-V
$$

\section{§ 4. Stable approximations}

In I and II we considered approximate equations for fairly long, fairly low waves. In these approximations the quantities $\eta=h-1$ and $v=\phi_{x}$ and the operator $\partial^{2} / \partial x^{2}=-k^{2}$ are supposed to be of order $\varepsilon$. It is then required that the equations are of Hamiltonian type and that the Hamiltonian is correct up till terms of the order $\varepsilon^{3}$. In 11 it was shown that these Hamiltonians always can be written as:

$$
\mathscr{H}=\int \mathrm{d} x \cdot\left[\frac{1}{2} \phi_{x} \cdot R \phi_{x}+\frac{1}{2} \eta^{2}+\frac{1}{2} \eta \phi_{x}^{2}+O\left(\varepsilon^{4}\right)\right],
$$

where the Fourier transform of the operator $R$ is given by

$$
\hat{R}=\frac{\operatorname{tgh} k}{k}=1-\frac{k^{2}}{3}+\frac{2 k^{4}}{15}+\ldots
$$

The last term in (1) implicates that there is a certain amount of freedom in choosing suitable simple approximations for $R$. Making no other use of this freedom we obtain:

$$
\mathscr{H}_{a}=\int \mathrm{d} x \cdot\left[\frac{1}{2} \phi_{x} R_{a} \phi_{x}+\frac{1}{2} \eta^{2}+\frac{1}{2} \eta \phi_{x}^{2}\right]
$$

which yields the Boussinesq type equations:

$$
\begin{gathered}
\eta_{t}=-\left(R_{a} \phi_{x}+\eta \phi_{x}\right)_{x} \\
\phi_{t}=-\left(\eta+\frac{1}{2} \phi_{x}^{2}\right) .
\end{gathered}
$$

Equation (4) shows that in all models of this kind $\eta$ is a conserved flux quantity. Equation (5) represents, up to the required order, the Bernoulli condition at the surface. It is seen from (5) that $\phi_{t}=O(\varepsilon)$.

In II it is observed that the simple approximation

$$
R_{a}=1+\frac{1}{3} \frac{\partial^{2}}{\partial x^{2}}
$$


which leads to a classical form of the Boussinesq equations, implies instability for short waves. It was shown that this can be repaired by taking e.g.

$$
R_{a}=R_{1}=\left(1-\frac{1}{3} \frac{\partial^{2}}{\partial x^{2}}\right)^{-1}
$$

which is a simple integral operator.

Although we are inclined to believe that the latter type of equations will be satisfactory in practice it must be conceded that they are not stable in any general sense. The danger of course lies in the term $\frac{1}{2} \eta \phi_{x}^{2}$. Whenever a solution could evolve in which the motion in the throughs of the waves were much stronger than elsewhere stability is not guaranteed.

In order to avoid this possible difficulty we look for an approximation for $\mathscr{H}$ which can be written as an integral over a sum of squares. This can be done by adding a term $\alpha \cdot \phi_{x}^{4}=O\left(\varepsilon^{4}\right)$ to the integral. Introducing a positive Hermitian operator $G$ by

$$
R=G^{2}
$$

we have:

$$
\mathscr{H}_{1}=\int \mathrm{d} x\left[\frac{1}{2}\left(G \phi_{x}\right)^{2}+\frac{1}{2} \eta^{2}+\frac{1}{2} \eta \phi_{x}^{2}+\alpha \phi_{x}^{4}\right],
$$

which yields (5) and

$$
\eta_{t}=-\left(G^{2} \phi_{x}+\eta \phi_{x}+4 \alpha \phi_{x}^{3}\right)_{x}
$$

as equations of motion. From (10) it is clear that the constant flux property has to be sacrified now.

In order to investigate the stability we rewrite (9) as:

$$
\begin{array}{r}
\mathscr{H}_{1}=\int \mathrm{d} x \cdot\left[\frac{1}{2}\left(G \phi_{x}\right)^{2}+\frac{1}{2}(1-\beta) \eta^{2}+\frac{1}{2} \beta\left(\eta+\frac{1}{2 \beta} \phi_{x}^{2}\right)^{2}+\right. \\
\left.+\left(\alpha-\frac{1}{8 \beta}\right) \phi_{x}^{4}\right] .
\end{array}
$$

When $\alpha$ is chosen to satisfy $\alpha>\frac{1}{8}$, a value of $\beta$ can be found such that $1>\beta>1 / 8 \alpha$, and $\mathscr{H}_{1}$ is in that case the sum of certain norms. From this it follows that the zero state of the model is stable in Lyapunov sense with respect to the metric

$$
\left\|G \phi_{x}\right\|^{2}+\|\eta\|^{2}+\left\|\phi_{x}^{2}\right\|^{2},
$$

where \|\| is the usual $L_{2}$-norm. 
The stability limit obviously is given by $\alpha=\frac{1}{8}, \beta=1$. The Hamiltonian then is:

$$
\mathscr{H}_{2}=\int \mathrm{d} x \cdot\left[\frac{1}{2}\left(G \phi_{x}\right)^{2}+\frac{1}{2}\left(\eta+\frac{1}{2} \phi_{x}^{2}\right)^{2}\right]
$$

If this Hamiltonian is stable it will be in a weaker sense. We will return to this question briefly in $\S 5$.

In II we also considered a slightly different approximation, viz.

$$
\mathscr{H}=\int \mathrm{d} x \cdot\left[\frac{1}{2}\left(G \phi_{x}\right)^{2}+\frac{1}{2} \eta^{2}+\frac{1}{2} \eta\left(G \phi_{x}\right)^{2}+O\left(\varepsilon^{4}\right)\right] .
$$

When a suitable approximation, e.g. $\hat{G}_{a}=\left(1+\frac{1}{6} k^{2}\right)^{-1}$, is used this Hamiltonian is stable for low, short waves. It can be stabilised throughout by adding a suitable fourth-order term. We consider here only the limit case and take:

$$
\mathscr{H}_{3}=\int \mathrm{d} x\left[\frac{1}{2}\left(G_{a} \phi_{x}\right)^{2}+\frac{1}{2}\left(\eta+\frac{1}{2}\left(G_{a} \phi_{x}\right)^{2}\right)^{2}\right] .
$$

This expression can be simplified somewhat by the canonical transformation

$$
w=G_{a} \phi_{x}, \quad \eta=-G_{a} \mu_{x}
$$

but the most symmetric form is obtained by using the mixed set of variables $\eta$ and $w$. In this way we find, proceeding as in II,

$$
\begin{gathered}
\eta_{t}-\frac{1}{6} \eta_{t x x}=-\frac{\partial}{\partial x} \frac{\partial E}{\partial w} \\
w_{t}-\frac{1}{6} w_{t x x}=-\frac{\partial}{\partial x} \frac{\partial E}{\partial \eta}
\end{gathered}
$$

where $E=\frac{1}{2}\left\{w^{2}+\left(\eta+\frac{1}{2} w^{2}\right)^{2}\right\}$.

These equations are of the "Hiden Hamiltonian" kind [7]. Putting:

$$
w=\rho-\sigma, \quad \eta=\rho+\sigma
$$

and neglecting $\sigma$ afterwards we find an B.B.M. like approximation for uni-directional waves, viz:

$$
\rho_{t}-\frac{1}{6} \rho_{t x x}=-\frac{\partial}{\partial x}\left(\rho+\frac{3}{4} \rho^{2}+\frac{1}{4} \rho^{3}\right)
$$

Another interesting special case of (1) is furnished by:

$$
\mathscr{H}_{4}=\int \mathrm{d} x \cdot\left[\frac{1}{2}\left(G \phi_{x}\right)^{2}+\frac{1}{2} \eta^{2}+\frac{1}{2}(G \eta)\left(G \phi_{x}\right)^{2}\right] .
$$


This can be simplified by the canonical transformation

$$
G \phi=\bar{\phi}, \quad \eta=G \bar{\eta}
$$

from which we obtain:

$$
\begin{gathered}
\mathscr{H}_{4}=\int \mathrm{d} x\left[\frac{1}{2} \bar{\phi}_{x}+\frac{1}{2} \bar{\eta} R \bar{\eta}+\frac{1}{2} R \bar{\eta} \cdot \bar{\phi}_{x}^{2}\right] \\
\bar{\eta}_{t}=-\left[\bar{\phi}_{x}(1+R \bar{\eta})\right]_{x} \\
\bar{\phi}_{t}=-\left[R \bar{\eta}+R \cdot \frac{1}{2} \bar{\phi}_{x}^{2}\right] .
\end{gathered}
$$

Introducing the variables:

$$
\bar{v}=\bar{\phi}_{x}, \quad \bar{\eta}=R \bar{\eta}
$$

the equations (19) take the form:

$$
\begin{aligned}
& R^{-1} \bar{\eta}_{t}=-[\bar{v}+\overline{\bar{\eta}} \bar{v}]_{x} \\
& R^{-1} \bar{v}_{t}=-\left[R^{-1} \overline{\bar{\eta}}+\frac{1}{2} \overline{\bar{v}}^{2}\right]_{x} .
\end{aligned}
$$

These equations are again of the hidden Hamilton form with

$$
\mathscr{H}=\int \mathrm{d} x\left[\frac{1}{2} \bar{v}^{2}+\frac{1}{2} \overline{\bar{\eta}} R^{-1} \overline{\bar{\eta}}+\frac{1}{2} \overline{\bar{\eta}} \bar{v}^{2}\right] .
$$

When we take the approximation (7):

$$
R=R_{1}, \quad R^{-1}=1-\frac{1}{3} \frac{\partial^{2}}{\partial x^{2}}
$$

the equations (21) correspond exactly to a set of equations investigated by Bona and Smith [3]. In this paper a number of rather remarkable results concerning the existence and behaviour of the solutions of (21) where derived. In considering the physical implications of these results one must keep in mind that the variables do not represent exactly the wave profile and the surface velocity. From (17) and (20) we infer:

$$
\bar{v}=G v, \quad \bar{\eta}=G \eta,
$$

which implies that the constant integral $\mathscr{H}$ indeed is the true energy $\mathscr{H}_{4}$. Consequently, the result that $\bar{\eta}$ is uniformly bounded, under certain conditions on the initial data, does not hold for the exact wave height $\eta$ : the boundedness of the first Sobolev norm $\|\bar{\eta}\|_{1}$ merely states that the $L_{2}$-norm of $\eta$ remains bounded, in agreement with the result obtained for the model described by $\mathscr{H}_{1}$ given in (9). 


\section{§5. Lagrangians}

Given an Hamiltonian the corresponding Lagrangian can be found when it is possible to express one of the variables in the time derivative of the other. Both forms of the theory are of course equivalent. Practical advantages and drawbacks of one or another formulation must be decided for each problem separately.

Equations of the kind considered in $\S 4$ turn out to have relatively simple Lagrangians only in terms of the variable $\phi$. They are found from the relation

$$
\mathscr{L}+\mathscr{H}=-\int \mathrm{d} x \cdot \eta \cdot \phi_{t}
$$

upon eliminating $\eta$ by means of the Bernoulli condition. Considering first the general Hamiltonian (4.1) we obtain:

$$
\mathscr{L}=\int \mathrm{d} x \cdot\left[\frac{1}{2} \phi_{t}^{2}-\frac{1}{2} \phi_{x} R \phi_{x}+\frac{1}{2} \phi_{t} \phi_{x}^{2}+O\left(\varepsilon^{4}\right)\right] .
$$

We observe that the third order term is linear in $\phi_{t}$. Therefore there is no third order term in the energy functional $E$, which is numerically equal to $\mathscr{H}$. We find:

$$
E=\int \mathrm{d} x \cdot\left[\frac{1}{2} \phi_{t}^{2}+\frac{1}{2} \phi_{x} R \phi_{x}+O\left(\varepsilon^{4}\right)\right],
$$

which, of course, is a constant of the motion. The equation of motion now reads:

$$
\phi_{t t}-R \phi_{x x}+\alpha \phi_{x} \phi_{t t}+\phi_{x x} \phi_{t}+O\left(\varepsilon^{4}\right)=0
$$

The most simple expressions for $\mathscr{L}$ result when the $O\left(\varepsilon^{4}\right)$ terms are dropped altogether. It is easily found that:

$$
\mathscr{L}_{2}=\int \mathrm{d} x \cdot\left[\frac{1}{2} \phi_{t}^{2}-\frac{1}{2} \phi_{x} R \phi_{x}+\frac{1}{2} \phi_{t} \phi_{x}^{2}\right]
$$

corresponds exactly to $\mathscr{H}_{2}$ as given by (4.12). From (4.14) we obtain:

$$
\mathscr{L}_{3}=\int \mathrm{d} x\left[\frac{1}{2} \phi_{t}^{2}-\frac{1}{2}\left(G \phi_{x}\right)^{2}+\frac{1}{2} \phi_{t} \cdot\left(G \phi_{x}\right)^{2}\right],
$$

where $G$ is any positive operator which satisfies

$$
G^{2}=R+O\left(\varepsilon^{4}\right)
$$

Therefore these simple Lagrangians lead to equations corresponding to the stability limits considered in $\S 4$. The corresponding energy integral is:

$$
E_{3}=\int \mathrm{d} x\left[\frac{1}{2} \phi_{t}^{2}+\frac{1}{2}\left(G \phi_{x}\right)^{2}\right] .
$$

By a suitable choice of $G, E_{2}$ - also can be written in this form. 
Once it has been decided to work within the Lagrangian framework transformations can be considered which simplify $\mathscr{L}$ without bothering whether the Hamiltonian equations also do improve under this transformation. As an example we consider a transformation which replaces the integro-differential equation (4) by a differential equation. We take:

$$
\mathscr{L}_{5}=\int \mathrm{d} x \cdot\left[\frac{1}{2} \phi_{t}^{2}-\frac{1}{2}\left(G \phi_{x}\right)^{2}+\frac{1}{2}\left(G \phi_{t}\right)\left(G \phi_{x}\right)^{2}\right] .
$$

The canonical equations resulting from $\mathscr{H}_{5}$ are more complicated than (4.4) and (4.5), we will not pay any attention to them.

We now use the approximation

$$
G=G_{1}=\left(1-\frac{1}{6} \frac{\partial^{2}}{\partial x^{2}}\right)^{-1}
$$

and introduce a new variable $\chi$ by

$$
\chi=G_{1} \phi, \quad \phi=\chi-\frac{1}{6} \chi_{x x} .
$$

In this way we find:

$$
\mathscr{L}_{5}=\int \mathrm{d} x\left[\frac{1}{2} \chi_{t}^{2}+\frac{1}{6} \chi_{t x}^{2}-\frac{1}{2} \chi_{x}^{2}+\frac{1}{2} \chi_{t} \chi_{x}^{2}\right],
$$

which leads to the differential equation:

$$
\chi_{t t}-\frac{1}{6} \chi_{x x t t}-\chi_{x x}+2 \chi_{x} \chi_{x t}+\chi_{x x} \chi_{t}=0
$$

and to the energy integral

$$
E_{5}=\int \mathrm{d} x\left[\frac{1}{2} \chi_{t}^{2}+\frac{1}{6} \chi_{x t}^{2}+\frac{1}{2} \chi_{x}^{2}\right], \quad \frac{\mathrm{d} E_{5}}{\mathrm{~d} t}=0 .
$$

From (12) we conclude that the present approximation is certainly stable in terms of the function $\chi$. A corresponding result is easily obtained for (7). As $G_{1}^{-1}$ is not bounded, stability in terms of $\phi$ is not warranted. Stated in more physical terms: when $\left|\phi_{x}\right|$ in a certain region is $O(1),\left|\phi_{x x}\right|$ extremely large, $\eta$ can be adapted in such a way that $\phi_{t}$ and $E_{3}$ are very small whereas the "true" energy (2.5) is finite. We do not believe that this circumstance would be a serious danger in practical applications of the equations. If this optimism should turn out to be unfounded the whole problem could be circumvented by using, in (2) or (4.1), an approximation for $R$ which is small but finite for large $k$. An example of such an approximation would be:

$$
\widehat{R}=\frac{1+\frac{1}{15} k^{2}}{1+\frac{2}{5} k^{2}}=1-\frac{1}{3} k^{2}+\frac{2}{15} k^{4}+\ldots
$$


This operator leads to more complicated equations. It has the additional advantage of reproducing the third term in (4.2).

This does not mean that the resultant equations are correct to the next order. Other terms of this order would appear due to the difference between $\phi$ and $\phi_{1}$ and to the approximation to be used for $T_{f}$ (compare II). It is possible to take all this into account and to write down a Hamiltonian which is correct up to terms of order $\varepsilon^{4}$. We will not do this here as the resultant equations are so complicated that their practical applicability seems very doubtful. Equation (10) corresponds to an equation given in a report by Valkering [8]. It was shown there that it furnishes a convenient approach to the problem of the interaction of two solitary waves. However, on first sight there is a discrepancy between Valkering's work and ours. The difficulty does not lie in equation (11) and the way it is used in dealing with the interaction problem, but in the interpretation of the variable $\chi$. Valkering derived (11) in the classical way by expanding $\phi(x, y)$ from the bottom upwards.

$$
\phi=f(x)-\frac{1}{2} y^{2} f_{x x}+\ldots,
$$

and eventually finds that $f(x, t)$ satisfies (11) and (12). In the present approximation this would mean that:

$$
\phi=\chi-\frac{1}{2} \chi_{x x}
$$

instead of (9). It turns out however that both (9) and (13) lead to (11) in the present approximation. There is a difference between the relation of $\eta$ and the conjugate variable $\chi$. Therefore Valkering's approach is perfectly valid provided the necessary care is used in calculating the wave profile from $\chi$. When a certain solution of (11) is considered the transformations (9), (13) or $\eta=\chi$ lead to slightly different velocity distributions and wave profiles, all of which are approximations to slightly different solutions of the exact equations.

\section{§ 6. A discrete model}

In some cases it is convenient, or at any rate instructive, to have a discrete model for some more or less complicated continuous problems. A very simple example of this is the relation between linear chains and longitudinal wave equations. In this section we will consider such a model for a Boussinesq type of equation.

A convenient starting point is furnished by the Lagrangian $\mathscr{L}_{3}$ as de- 
fined by (5.6). Denoting the real antisymmetric operator $G(\partial / \partial x)$ by $A$ we have:

$$
\mathscr{L}_{3}=\int \mathrm{d} x\left[\frac{1}{2} \phi_{t}^{2}-\frac{1}{2}(A \phi)^{2}+\frac{1}{2} \phi_{t}(A \phi)^{2}\right] .
$$

In the same way as in the foregoing section we derive the energy integral:

$$
E_{3}=\int \mathrm{d} x\left[\frac{1}{2} \phi_{t}^{2}+\frac{1}{2}(A \phi)^{2}\right]
$$

and the Hamiltonian:

$$
\mathscr{H}_{3}=\int \mathrm{d} x\left[\frac{1}{2}(A \phi)^{2}+\frac{1}{2}\left(\eta+\frac{1}{2}(A \phi)^{2}\right)^{2}\right] .
$$

Taking $(\eta, \phi)$ as a pair of conjugate variables $(q, p)$, we derive the canonical equations. Introducing the velocity-like variable $w$ by

$$
w=A \phi=G \phi_{x}=G v,
$$

we write these equations as:

$$
\begin{aligned}
& \eta_{t}=-A\left(w+\eta w+\frac{1}{2} w^{3}\right) \\
& w_{t}=-A\left(\eta+\frac{1}{2} w^{2}\right),
\end{aligned}
$$

which are quite analogous to (4.15).

We now replace the continuous variables $\eta(x), w(x)$ by discrete functions $\eta_{n}, w_{n}$. These correspond to $\eta, w$ when a certain step length of order 1 is taken. The exact value of this step length or the period of the chain remains to be chosen. The only information required is now a suitable discrete representation of the operator $A$. This is easily found by considering the eigenfunctions or eigenvectors.

In the continuous case the eigenfunctions are of course exp ikx. As $A=G(\partial / \partial x)$ and $G^{2}=R$ we obtain, using (4.2)

$$
A \cdot \exp \mathrm{i} k x=\left\{\mathrm{i} k\left(1-\frac{k^{2}}{6}\right)+O\left(k^{5}\right)\right\} \exp \mathrm{i} k x .
$$

In order to reproduce this spectrum of $A$ it is convenient to fix the step length at 1 exactly. This means that this step length is the same as the unperturbed waterdepth in the continuous problem to be modeled. The corresponding eigenvectors then are $e_{n}=\operatorname{exp~ikn}$. We now define:

$$
A e_{n}=\frac{e_{n+1}-e_{n-1}}{2}
$$

and find:

$$
A e_{n}=\mathrm{i} \sin k \cdot e_{n}=\mathrm{i} k\left(1-\frac{k^{2}}{6}\right) \cdot e_{n}+O\left(k^{5}\right) .
$$


In this way we obtain a discrete representation of (5) in the form:

$\dot{\eta}_{n}=\frac{1}{2}\left(w_{n-1}+\eta_{n-1} w_{n-1}+\frac{1}{2} w_{n-1}^{3}-w_{n+1}-\eta_{n+1} w_{n+1}-\frac{1}{2} w_{n+1}^{3}\right)$

$\dot{w}_{n}=\frac{1}{2}\left(\eta_{n-1}+\frac{1}{2} w_{n-1}^{2}-\eta_{n+1}-\frac{1}{2} w_{n+1}^{2}\right)$.

It is possible to derive these equations from the discrete version of (1):

$$
\mathscr{L}_{d}=\sum_{n}\left\{\frac{1}{2} \dot{\phi}_{n}^{2}+\frac{1}{8} \dot{\phi}_{n}\left(\phi_{n+1}-\phi_{n-1}\right)^{2}-\frac{1}{8}\left(\phi_{n+1}-\phi_{n-1}\right)^{2}\right\},
$$

but we will not give this, essentially very simple, derivation here.

We now have to consider the stability of our model in some region around the equilibrium state. This state is represented by $\eta_{n}=0, w_{n}=0$, $\phi_{n}=$ constant. Observing that the energy integral (2) now becomes:

$$
\begin{aligned}
E_{d}=\sum_{n}\left(\frac{1}{2} \dot{\phi}_{n}^{2}+\frac{1}{2} w_{n}^{2}\right)=\sum_{n}\left[\frac{1}{2} \dot{\phi}_{n}^{2}+\frac{1}{8}\left(\phi_{n+1}-\phi_{n-1}\right)^{2}\right]= \\
=\sum_{n}\left[\frac{1}{2}\left(\eta_{n}+\frac{1}{2} w_{n}^{2}\right)^{2}+\frac{1}{2} w_{n}^{2}\right]
\end{aligned}
$$

the stability is obvious.

The solution of (7) from given initial data directly yields the wave profile $\eta_{n}(t)$. The velocity $v_{n}$ can be found from a numerical representation of (4). Proceeding in the same way as in deriving (6) we would obtain:

$$
v_{n}=\frac{8 w_{n}-w_{n+1}-w_{n-1}}{6}
$$

which then completes our solution.

In connection with the stability of the model one final remark will be made. At first sight the use of the approximation

$$
\hat{A}=\mathrm{i} k\left(1-\frac{k^{2}}{6}\right)+O\left(k^{5}\right)
$$

might suggest that we essentially used the approximation:

$$
G=1+\frac{1}{6} \frac{\partial^{2}}{\partial x^{2}}
$$

which, in the continuous model, leads to rather weird behaviour of short waves. The reason that we now obtained a more useful approximation lies of course in the entirely different behaviour of discrete and continuous models for short waves. The unit step length means that the spurious occurrence of waves with $k>\pi$ is avoided. This is obvious when 
we consider the linearised equations. Dropping the third order term from $\mathscr{L}_{3}$ and using (4) and (9) we obtain the dispersion relation

$$
\omega^{2}=k^{2}\left(1-\frac{k^{2}}{6}\right)^{2}
$$

which means that the fase- and group velocities are unbounded and of order $k^{2}$ for short waves. When the second term in $\mathscr{L}_{d}$ is struck we obtain the equations

$$
\ddot{\phi}_{n}=\frac{\phi_{n+2}-2 \phi_{n}+\phi_{n-2}}{4}
$$

which leads to the dispersion relation

$$
w^{2}=\frac{1}{2}(1-\cos 2 k)=\sin ^{2} k \text {. }
$$

This implies that both velocities are bounded now. We are inclined, as stated before, to believe that the marginal stability of $\mathscr{H}_{2}$ or $\mathscr{H}_{3}$ will in practice be sufficient when suitable numerical procedures for solving initial value problems for the resulting equations of motion are used. The stability of the equations (7) points in this direction.

\section{§ 7. A stable approximation for uneven bottom}

In this section we consider long waves over a bottom profile of small slope. The aim is to derive a set of equations like (4.5) and (4.10), furnishing a stable approximation for fairly long, fairly low waves.

We start from equations (2.1) to (2.4) in a slightly modified form:

$$
\left.\begin{array}{c}
\Phi_{x x}+\Phi_{y y}=0 \\
\Phi_{y}+h_{x} \Phi_{x}=0 \text { at } y=-h(x) \\
\eta_{t}=\Phi_{y}-\eta_{x} \Phi_{x} \\
\Phi_{t}=-\frac{1}{2}\left(\Phi_{x}^{2}+\Phi_{y}^{2}\right)-\eta
\end{array}\right\} \text { at } y=\eta(x, t) .
$$

In these equations $y=-h$ is the bottom, $y=\eta$ the wave profile. It can be shown that the canonical theorem is valid for these equations. The quantities $\phi(x, t)=\Phi(x, \eta, t)$ and $\eta$ are a pair of canonically conjugate variables. We suppose that $h(x)>\delta>0$.

We now need a suitable approximation for the energy. Following the procedure of II we split the kinetic energy in a part corresponding to $0>y>-h$ and a small rest term. This rest can be approximated in the same way as in II. In this way we obtain

$$
E=\int \mathrm{d} x\left[\frac{1}{2} \phi_{0}^{\prime} \psi_{0}+\frac{1}{2} \eta \phi_{0}^{\prime 2}+\frac{1}{2} \eta^{2}\right],
$$


where we wrote:

$$
\phi_{0}=\Phi(x, 0, t), \quad \psi_{0}=\Psi(x, 0, t) .
$$

$\Psi$ is the stream function corresponding to $\Phi$. Differentiation of $y$-independent quantities with respect to $x$ is denoted by a dash.

We now formally expand $\Phi$ and $\Psi$ :

$$
\begin{aligned}
& \Phi=\phi_{0}-y \psi_{0}^{\prime}-\frac{1}{2} y^{2} \phi_{0}^{\prime \prime}+\frac{1}{6} y^{3} \psi_{0}^{\prime \prime} \ldots \\
& \Psi=\psi_{0}+y \phi_{0}^{\prime}-\frac{1}{2} y^{2} \psi_{0}^{\prime \prime}-\frac{1}{6} y^{3} \phi_{0}^{\prime \prime} \ldots,
\end{aligned}
$$

and observe that from (2) it is allowed to choose

$$
\Psi(x,-h)=0 .
$$

Combining (7) and (8) and solving formally by iteration we obtain:

$$
\psi_{0}=h \psi_{0}^{\prime}+\frac{1}{2} h^{2}\left(h \phi_{0}^{\prime}\right)^{\prime \prime}-\frac{1}{6} h^{3} \phi_{0}^{\prime \prime \prime}+\ldots
$$

Substituting (9) into (5) we find, after some integrations by part:

$$
E=\int \mathrm{d} x\left[\frac{1}{2}\left(h+h^{2} h^{\prime \prime}+\eta\right) \phi_{0}^{\prime 2}+\frac{1}{2} \eta^{2}-\frac{1}{6} h^{3} \phi_{0}^{\prime \prime 2} \ldots\right]
$$

We now have to decide about the order of magnitude of the quantity $h h^{\prime \prime}$. In our models we always took $\phi^{\prime}$ and $\eta$ to be of order $\varepsilon$. The operator $\partial / \partial x$, applied to $\eta$ or $\phi^{\prime}$ essentially is of order $\varepsilon^{\frac{1}{2}}$, or $k^{2}=O(\varepsilon)$ in Fourier language. Obviously it is consistent to take $h=O(1)$. When the dominant wave length of surface and bottom profile are of the same order, $h h^{\prime \prime}=O(\varepsilon)$. If, however, the bottom slope is very small we could take $h h^{\prime \prime}=O\left(\varepsilon^{2}\right)$ and drop the term with $h^{\prime \prime}$. We settle for the second, much simpler, choice. As it is easily seen that here again:

$$
\phi^{\prime}-\phi_{0}^{\prime}=O\left(\varepsilon^{3}\right)
$$

we have:

$$
\mathscr{H}=\int \mathrm{d} x\left[\frac{1}{2} h \phi^{\prime 2}-\frac{1}{6} h^{3} \phi^{\prime \prime 2}+\frac{1}{2} \eta \phi^{\prime 2}+\frac{1}{2} \eta^{2}+O\left(\varepsilon^{4}\right)\right] .
$$

This Hamiltonian is a good approximation for long waves; it is however of the classical Boussinesq type and therefore it has to be stabilized in some way by a suitable choice of terms $O\left(\varepsilon^{4}\right)$. In this connection it turns out that it is advantageous to change variables. We write:

$$
\begin{aligned}
h \frac{\partial}{\partial x} & =\frac{\partial}{\partial z}, \quad z=\int_{0}^{x} \frac{\mathrm{d} x}{h} \\
\phi(x) & =\bar{\phi}(z)
\end{aligned}
$$




$$
\begin{aligned}
& h(x)=\bar{h}(z) \\
& \eta(x)=\frac{\xi(z)}{\bar{h}(z)} .
\end{aligned}
$$

It can be shown [9] that this is a canonical transformation, $\bar{\phi}$ and $\xi$ are again a pair of conjugate variables. Performing these substitutions and dropping a number of small terms (remembering that $\mathrm{d} h / \mathrm{d} z=O(\varepsilon)$ ), we eventually arrive at:

$$
\mathscr{H}=\int \mathrm{d} z \cdot\left[\frac{1}{2} \bar{\phi}_{z}^{2}-\frac{1}{6} \bar{\phi}_{z z}^{2}+\frac{1}{2} \frac{\xi}{\bar{h}^{2}} \bar{\phi}_{z}^{2}+\frac{1}{2} \frac{\xi^{2}}{\bar{h}}+O\left(\varepsilon^{4}\right)\right]
$$

In order to stabilize (12) we introduce the operator

$$
\bar{R}=\left(1-\frac{1}{3} \frac{\partial^{2}}{\partial z^{2}}\right)^{-1}
$$

which again is a simple integral operator in $z$. A suitable Hamiltonian then is:

$$
\mathscr{H}=\int \mathrm{d} z \cdot\left[\frac{1}{2} \bar{\phi}_{\mathrm{z}} \bar{R} \bar{\phi}_{\mathrm{z}}+\frac{1}{2 \bar{h}}\left(\xi+\frac{1}{2} \frac{\bar{\phi}_{z}^{2}}{\bar{h}}\right)^{2}\right],
$$

although of course many other expressions could be used.

From (13) we obtain the Bernoulli equation in the form:

$$
\bar{\phi}_{t}=-\left(\frac{\xi}{\bar{h}}+\frac{1}{2} \frac{\bar{\phi}_{z}^{2}}{\bar{h}^{2}}\right)
$$

Therefore this model has the Lagrangian:

$$
\mathscr{L}=\int \mathrm{d} z \cdot\left[\frac{\bar{h}}{2} \bar{\phi}_{t}^{2}-\frac{1}{2} \bar{\phi}_{z} \bar{R} \bar{\phi}_{z}+\frac{1}{2 \bar{h}} \bar{\phi}_{t} \bar{\phi}_{z}^{2}\right] .
$$

The energy integral now is:

$$
E=\int \mathrm{d} z\left[\frac{1}{2} \bar{\phi}_{\mathrm{z}} \bar{R} \bar{\phi}_{\mathrm{z}}+\frac{\vec{h}}{2} \bar{\phi}_{t}^{2}\right]
$$

Therefore this model is stable in the sense discussed in $\S 5$.

Received 30 November 1976 


\section{REFERENCES}

[1] Broer, L. J. F., Appl. Sci. Res. 29 (1974) 430.

[2] Broer, L. J. F., Appl. Sci. Res. 31 (1975) 377.

[3] Bona, J. L. and R. Smith, Math. Proc. Camb. Phil, Soc. 79 (1976) 167.

[4] Benjamin, T. B., Lectures on Nonlinear Wave Motion, p. 3-47 in A. C. Newel, Ed., Nonlinear Wave Motion, Lectures in Applied Math. Vol. 15 (1974) A.M.S.

[5] Benjamin, T. B. and J. J. Mahony, J. Fluid Mech. 49 (1971) 385.

[6] BRoER, L. J. F. and S. C. M. BACKerRA, Appl. Sci. Res. 32 (1976) 495.

[7] Broer, L. J. F., Physica 79A (1975) 583.

[8] Valkering, T. P., Physical Communications, Twente University of Technology, Vol. 8, nr. 1, August 1976.

[9] Broer, L. J. F. and J. A. Kobussen, Physica 61 (1972) 275. 